\title{
Impact of Transesophageal Echocardiography on Clinical Management of Patients Over Age 50 With Cryptogenic Stroke and Normal Transthoracic Echocardiogram
}

\author{
Brian Marino, DO , Abhishek Jaiswal, MD², Seth Goldbarg, MD³, Gary L. Bernardini, MD, PhD4, Todd Kerwin, MD ${ }^{5 *}$
}

\begin{abstract}
${ }^{1}$ Division of Cardiology, Department of Internal Medicine, New York Hospital Queens/Weill Medical College-Cornell University, Flushing, New York; 2Tulane University Heart and Vascular Institute, Tulane School of Medicine, New Orleans, Louisiana; ${ }^{3}$ Division of Cardiology, New York Hospital Queens/Weill Medical College-Cornell University, Flushing, New York; ${ }^{4}$ Department of Neurology, New York Hospital Queens/Weill Medical CollegeCornell University, Flushing, New York; ${ }^{5}$ Non-Invasive Cardiology and Cardiology Fellowship Program, Division of Cardiology, New York Hospital Queens/Weill Medical College-Cornell University, Flushing, New York.
\end{abstract}

BACKGROUND: The utility of transesophageal echocardiography (TEE) subsequent to a normal transthoracic echocardiogram (TTE) in older patients with an unexplained stroke is uncertain.

METHODS: Two hundred sixty-three consecutive patients over the age of 50 years hospitalized with a clinical stroke confirmed with brain magnetic resonance imaging and a normal TTE were retrospectively analyzed. Patients with atrial fibrillation, atrial flutter, or any other causative reason for stroke were excluded. TEE was analyzed for findings that could explain the etiology of stroke as well as findings that would change therapy based on current guidelines.

RESULTS: Baseline characteristics included a mean age of 66.7 years (range, $50-91$ years); $42.5 \%$ of patients were female. A possible etiology of stroke was discovered by TEE in $111(42.2 \%)$ patients and included 1 or more of the following: complex plaque of the ascending aorta or arch, patent foramen ovale, atrial septal aneurysm, both atrial septal aneurysm and patent foramen ovale, or spontaneous contrast. Only 1 patient $(0.4 \%)$ had a finding that changed immediate management, which was a thrombus in the left atrial appendage for which anticoagulation was prescribed. Follow-up was available at 6 months on 85 patients, of whom $13(15 \%)$ had been discovered to have developed atrial fibrillation.

CONCLUSION: In our study population, when performed subsequent to a normal TTE in patients aged $>50$ years with cryptogenic stroke, TEE demonstrated a high diagnostic value, but had minimal incremental effect on patient management. Journal of Hospital Medicine 2016;11:95-98. (C) 2015 Society of Hospital Medicine
Specific transesophageal echocardiography (TEE) findings associated with stroke include cardiac thrombi (particularly left atrial appendage [LAA]), left atrial spontaneous echo contrast, interatrial septal anomalies (particularly patent foramen ovale [PFO]), and atheromatous disease of the aorta. In younger patients (aged $<50$ years) with stroke of uncertain etiology, TEE is often recommended because of reported higher yield than transthoracic echocardiogram (TTE), particularly in detecting PFO or atrial septal aneurysm (ASA). ${ }^{1}$

Aside from oral anticoagulation in patients with an intracardiac thrombus, current guidelines and scientific evidence do not support specific therapeutic interventions for the other TEE findings. For example, the most effective therapy for stroke prevention with findings of aortic arch plaque remains uncertain. In addition, the very rare patient presenting with stroke from

*Address for correspondence and reprint requests: Todd Kerwin, MD, New York Hospital Queens, WA 200, Division of Cardiology, 56-45 Main Street, Flushing, NY 11355; Telephone: 718-670-1130;

Fax: 718-661-7708; E-mail: tck9002@nyp.org

Additional Supporting Information may be found in the online version of this article.

Received: May 1, 2015; Revised: July 29, 2015; Accepted: August 2, 2015

2015 Society of Hospital Medicine DOI 10.1002/jhm.2484

Published online in Wiley Online Library (Wileyonlinelibrary.com). a cardiac tumor, which is generally visible on TTE, might benefit from surgical removal. ${ }^{2}$

We sought to examine the benefit of performing TEE after a normal TTE in patients over age 50 years admitted with a stroke of uncertain etiology. We hypothesized that there would be minimal change in management based on TEE findings after a normal TTE in older patients hospitalized with an unexplained stroke.

\section{METHODS}

Over a 4-year period from 2009 to 2012, all patients over the age of 50 years admitted to our communitybased teaching hospital with a primary diagnosis of ischemic stroke were identified and retrospectively screened by review of our institutional echocardiography database during this time period. Stroke diagnosis had to be confirmed with acute or subacute ischemia on brain magnetic resonance imaging. Patients with an indication for anticoagulation or who had a known history of atrial fibrillation or flutter were excluded. Patients were monitored with continuous telemetry during hospital admission and were also excluded if they developed atrial fibrillation or flutter after admission. Additionally, patients were excluded if a neurologist-directed evaluation revealed another etiology for the stroke. 
A TTE acquired in all patients was performed according to Intersocietal Commission for the Accreditation of Echocardiography Laboratories standards and included 2-dimensional, color Doppler, continuous wave, and pulse wave data. Images were obtained in the parasternal long and short axis, apical 4-chamber, 2-chamber, and long axis views. An abnormal TTE was defined as a study with a prosthetic valve, abnormal left ventricular (LV) systolic function, an intracardiac mass, intracardiac shunt, or severe valvular heart disease, as these significant findings may explain stroke.

Standardized TEE images were obtained with midesophageal 4-chamber, mitral commissural, 2-chamber, long axis, ascending aorta long axis, aortic valve short axis, right ventricular inflow-outflow, and bicaval views. Detailed multiplanar evaluation of the LAA was performed. If no interatrial shunt was visualized with color flow Doppler in the bicaval view, agitated intravenous saline was administered for further evaluation. Additional standard images were obtained of the descending aorta and aortic arch in the short and long axis. Transgastric images were obtained when feasible or necessary.

The study was submitted to our institutional review board. As no patient identifiers were stored, and we used previously existing data from an institutional echocardiography database to conduct the study, it was determined to be exempt.

Statistical analysis was performed by recording the prevalence of each potential cardiac source of embolism.

\section{RESULTS}

Of the 853 consecutive patients screened, 456 were excluded because of atrial fibrillation, atrial flutter, or another etiology of stroke. An additional 134 patients were excluded with an abnormal TTE or if a TEE was not performed. The remaining 263 patients were analyzed based on TEE findings (Figure 1).

The mean age was 66.7 years (range, 50-91 years), and $42.5 \%$ were female. A possible etiology of stroke (Table 1) discovered included complex plaque of the ascending aorta or arch 44/263 (16.7\%), PFO 18/263 $(6.8 \%)$, atrial septal aneurysm $25 / 263(9.5 \%)$, and both ASA and PFO in 11/263 (4.2\%), and spontaneous contrast was seen in the left atrium or LAA in 13/ $263(4.9 \%)$ patients. One patient had a thrombus in the LAA for which anticoagulation was prescribed. No other intracardiac masses were identified.

Overall, $42.6 \%$ of patients had a TEE finding which could explain the etiology of stroke or transient ischemic attack (TIA), but only 1 patient $(0.4 \%)$ had a finding that changed therapy. Follow-up was available at 6 months for 85 patients, and $13(15 \%)$ of these patients had been discovered to develop atrial fibrillation in the interim.

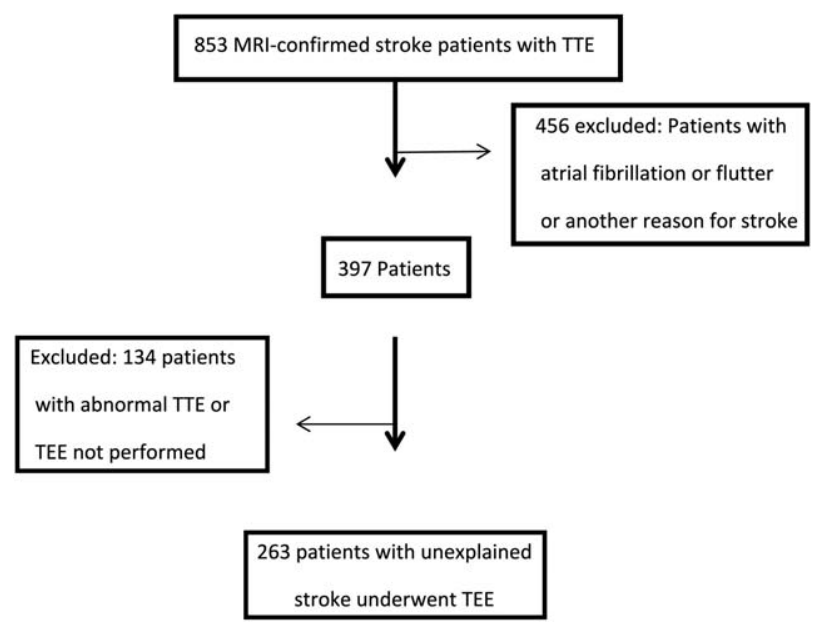

FIG. 1. Flowchart for identification of transesophageal echocardiography (TEE) analysis. Abbreviations: MRI, magnetic resonance imaging; TTE, transthoracic echocardiogram.

\section{DISCUSSION}

Our study retrospectively analyzed the utility of TEE in patients over age 50 years admitted with ischemic stroke without a clear etiology. We found that TEE provides significant incremental diagnostic benefit as compared to TTE in identifying a possible etiology of stroke in these patients. This is consistent with prior studies showing a high diagnostic yield of TEE in patient with ischemic stroke of uncertain etiology. ${ }^{3}$ However, in our study, based on current guidelines, virtually none of these findings directly altered patient management.

The 2014 guidelines for secondary stroke prevention recommend antiplatelet and statin therapy (in addition to lifestyle modification, smoking cessation, and blood glucose and blood pressure control) as a standard medical regimen in patients with stroke or TIA of uncertain etiology. The finding of aortic arch atheroma does not warrant supplementary treatment in addition to an antiplatelet and statin according to current guidelines. Atherosclerosis of the aortic arch is an important source of cerebral embolism, particularly in cases where plaque is $>4 \mathrm{~mm}$ in size. ${ }^{4} \mathrm{~A}$ recent study by Amarenco et al., comparing efficacy of

\begin{tabular}{lc}
\hline \multicolumn{2}{l}{ TABLE 1. Potential Cardiovascular Sources of } \\
Embolism by Transesophageal Echocardiogram \\
in 263 Patients \\
\hline Potential Source & No. (\%) \\
\hline Atrial septal aneurysm & $25(5.3 \%)$ \\
Patent foramen ovale & $18(2.7 \%)$ \\
Atrial septal aneurysm and patent foramen ovale & $11(4.2 \%)$ \\
Complex aortic plaque & $44(16.7 \%)$ \\
Spontaneous contrast & $13(4.9 \%)$ \\
Left atrial appendage thrombus* & $1(0.4 \%)$ \\
Total & $112(42.6 \%)$ \\
\hline
\end{tabular}

NOTE: 'This was the only finding on transesophageal echocardiography that changed management. Anticoagulation was prescribed. 
combined antiplatelet therapy (clopidogrel and aspirin) to warfarin in recurrent stroke prevention in patients with $>4 \mathrm{~mm}$ aortic arch plaque, showed nonsignificant reduction in rate of recurrent stroke with dual antiplatelet therapy. ${ }^{5}$ However, optimal therapy for these patients still remains uncertain beyond standard stroke-prevention treatment. Although there are emerging data on therapeutic options in patients with complex atheroma, there is currently no specific guideline-recommended therapy or consensus among stroke neurologists. Potentially, if an individual practitioner had a strong feeling on therapeutic modifications based on the presence of complex aortic arch atheroma, the TEE would have value to their patient. However, in our study, which had a prevalence of $16.8 \%$ of complex plaque of the ascending aorta or arch, there were no therapeutic changes based on this finding. This reinforces the limited value of this test that we observed in our study population.

Anticoagulation has not been shown to be superior to aspirin in patients with PFO (with or without ASA), and recent studies showed no benefit of procedural PFO closure compared to best medical management for stroke prevention (Randomized Evaluation of Recurrent Stroke Comparing PFO Closure to Established Current Standard of Care Treatment [RESPECT], Evaluation of the STARFlex Septal Closure System in Patients with a Stroke and/or Transient Ischemic Attack due to Presumed Paradoxical Embolism through a Patent Foramen Ovale [CLOSURE I]). ${ }^{6,7}$ However, a patient with a PFO and deep vein thrombosis would benefit from anticoagulation and consideration of PFO closure. ${ }^{8}$ This rare entity could be excluded with a simple lower extremity duplex without the need for a TEE, which does come with a small risk of complications related to anesthesia and local oropharyngeal trauma as well as discomfort to the patient and increased cost. Spontaneous echo contrast is not an independent indication for anticoagulation. If spontaneous contrast were associated with mitral stenosis and an embolic event, then anticoagulation would be indicated. ${ }^{9}$ Mitral stenosis is easily diagnosed with TTE.

LAA or left atrial thrombus is the predominant finding exclusive to TEE that would change management for secondary stroke prevention, specifically anticoagulation. Fifteen studies representing over 3000 patients in a 2014 meta-analysis reported the prevalence of left atrial or LAA thrombus in patients aged $\geq 55$ years with a cryptogenic stroke to be $4 \%$, with a range in the studies of $0 \%$ to $21.2 \% .^{3}$ The wide range of prevalence of this finding is likely related to the prevalence of known atrial arrhythmias or structural heart disease in the population of patients included in the analysis. Left atrial or LAA thrombus in the absence of systolic dysfunction, severe valve disease, or known atrial fibrillation is exceedingly uncommon $(0.3 \%) .{ }^{10}$ It is likely that the few patients with left atrial or LAA thrombus without 1 of these conditions probably has undiagnosed paroxysmal atrial fibrillation. In previous studies that showed a high prevalence of left atrial or LAA thrombus, there was no mention of the presence or absence of LV dysfunction or severe valve disease in patients with left atrial or LAA thrombus. Additionally, these studies only required a 12-lead electrocardiogram or did not specify the presence or duration of continuous rhythm monitoring. ${ }^{11-14}$ Several of the studies with high incidence of left atrial or LAA thrombus specifically stated that some of these patients were known to have atrial fibrillation. ${ }^{11,13}$

Approximately $8 \%$ of patients admitted with stroke are found to have atrial fibrillation only after admission with continuous electrocardiogram monitoring. The detection rate is nearly half if monitoring is limited to 24 hours instead of several days. Overall, detection rates of atrial fibrillation following stroke are relatively low during initial hospitalization. ${ }^{15}$ More intense monitoring for atrial fibrillation in patients with a stroke of uncertain etiology with the use of a subcutaneous implantable cardiac monitor increases the detection rate to $12.4 \%$ at 1 year, and increases with longer monitoring time. ${ }^{16}$ Therefore, identification of older stroke patients without significant stroke risk factors may be candidates for longerterm cardiac monitoring to increase yield for detection of atrial fibrillation. Currently, continuous electrocardiographic monitoring of patients for the duration of their hospitalization and up to 30 days afterward is recommended. ${ }^{8}$

Our study differs from prior studies that showed a much higher prevalence of LAA or left atrial thrombus in 2 important ways. Patients with severe valve disease or LV dysfunction were excluded on the basis of TTE. Additionally, our patients underwent continuous electrocardiographic monitoring for the duration of their hospitalization and were excluded with a prior history or newly discovered atrial fibrillation or flutter. Our intention was to examine the value of adding TEE when no other etiology of stroke was identified. Value can be defined as healthcare outcomes achieved per dollar spent. Our study was not designed to look at long-term outcomes; rather, we used immediate change in patient management as a surrogate.

There are several limitations to our study that must be noted. This was a single-center study potentially creating a bias as less stringent selection of patients undergoing TEE may be the practice at other institutions. This analysis was retrospective; therefore, there may have been bias as to which patients were selected to undergo TEE. Additionally, stroke subtype was not specified, and the pretest probability of a cardioembolic source differs based on subtype. Last, we focused this study on immediate changes in clinical management prompted by TEE results, and did not assess patient perceptions of TEE value related to 
enhanced knowledge about the etiology of their stroke; this area represents an opportunity for further research.

\section{CONCLUSIONS}

TEE provides a substantial increase in possible explanation of stroke etiology in patients over age 50 years admitted with a stroke of uncertain cause and a normal TTE. However, there is minimal incremental value in regard to change in therapeutic management in these patients. In a time of increased focus on providing cost effective healthcare, our findings suggest that the need for TEE in this stroke population should be more closely examined.

Disclosure: Nothing to report.

\section{References}

1. Rettig TCD, Bouma BJ, van den Brink RBA. Influence of transesophageal echocardiogram on therapy and prognosis in young patients with TIA or ischemic stroke. Neth Heart J. 2009;17:373-377.

2. Engberding R, Daniel WG, Erbel R, et al. Diagnosis of Heart Tumors by Transesophageal Echocardiography: a multicentre study in 154 patients. Eur Heart J. 1993;14:1223-1228.

3. McGrath ER, Paikin JS, Motlagh B, Salehian O, Kapral MK, O'Donnell MJ. Transesophageal echocardiography in patients with cryptogenic ischemic stroke: a systematic review. Am Heart J. 2014; 168:706-712.

4. Tunick PA, Perez JL, Kronzon I. Protruding atheromas in the thoracic aorta and systemic embolization. Ann Intern Med. 1991;115:423427.

5. Amarenco P, Davis S, Jones EF, et al.; The Aortic Arch Related Cerebral Hazard Trial Investigators. Clopidogrel plus aspirin versus warfarin in patients with stroke and aortic arch plaques. Stroke. 2014; 45:1248-1257.

6. Carroll JD, Saver JL, Thaler DE, et al.; RESPECT Investigators. Closure of patent foramen ovale versus medical therapy after cryptogenic stroke. N Engl J Med. 2013;368:1092-1100.

7. Furlan AJ, Reisman M, Massaro J, et al.; CLOSURE I Investigators. Closure or medical therapy for cryptogenic stroke with patent foramen ovale. N Engl J Med. 2012;366:991-999.

8. Kernan WN, Ovbiagele B, Black HR, et al. Guidelines for the prevention of stroke in patients with stroke and transient ischemic attack: a guideline for healthcare professionals from the American Heart Association/American Stroke Association. Stroke. 2014;45(7): 2160-2236.

9. Nishimura RA, Otto CM, Bonow RO, et al. 2014 AHA/ACA guideline for the management of patients with valvular heart disease. J Am Coll Cardiol. 2014; 63:e57-e185.

10. Agmon Y, Khandheria BK, Gentile F, Seward JB. Clinical and echocardiographic characteristics of patients with left atrial thrombus and sinus rhythm: experience in 20643 consecutive transesophageal echocardiographic examinations. Circulation. 2002;105(1):27-31.

11. Labovitz AJ, Camp A, Castello R, et al. Usefulness of transesophageal echocardiography in unexplained cerebral ischemia. Am J Cardiol. 1993;72:1448-1452.

12. Mattioli AV, Aquilina M, Bonetti L, Oldani A, Longhini C, Mattioli G. Transesophageal echocardiography in patients with recent stroke and normal carotid arteries. Am J Cardiol. 2001;88:820-823.

13. de Bruijn SF, Agema WR, Lammers GJ, et al. Transesophageal echocardiography is superior to transthoracic echocardiography in management of patients of any age with transient ischemic attack or stroke. Stroke. 2006;37:2531-2534.

14. Buser PT, Zuber M, Rickenbacher P, Erne P, Jenzer H, Burckhardt D. Age-dependent prevalence of cardioembolic sources detected by TEE: diagnostic and therapeutic implications. Echocardiography. 1997;14: 597-606.

15. Rizos T, Guntner J, Jenetzky E, et al. Continuous stroke unit electrocardiographic monitoring versus 24-hour Holter electrocardiography for detection of paroxysmal atrial fibrillation after stroke. Stroke. 2012;43:2689-2694.

16. Sanna T, Diener HC, Passman RS, et al.; CRYSTAL AF Investigators. Cryptogenic stroke and underlying atrial fibrillation. N Engl J Med. 2014;370(26):2478-2486. 